\title{
Defining the Scope of Your Evaluation
}

\author{
Elizabeth Wood \\ Indiana University-Purdue University Indianapolis
}

Contact information:

Elizabeth (Elee) Wood

IUPUI Museum Studies Program

Cavanaugh Hall 419

425 University Blvd.

Indianapolis, IN 46202

eljwood@iupui.edu

(317) 274-7332

This is the author's manuscript of the article published in final edited form as:

Wood, E. (2015). Defining the Scope of Your Evaluation. Journal of Museum Education, 40(1), 13-19. http://dx.doi.org/10.1080/10598650.2015.11510828 
Getting started with an evaluation process can be a daunting task, but it need not be full of complicated theories and processes. A successful evaluation is built on clear, thoughtful, and focused questions that can support improvement, use, and application of new ideas. One of the most challenging aspects of conducting evaluations is finding the right overarching evaluation question to guide the work. What it all boils down to is what you want to know and what you want to do with what you find. When the purpose of the evaluation process is clear, it will provide the evaluator with a good sense of what information can answer your questions, and helps frame the scope of the project as a whole. Knowing the scope of your evaluation project will help you get a sense of the resources (time, money, and people) needed.

As easy as this may seem, generating the question and understanding the scope of the evaluation is the first place where problems can happen. Too often, the need to conduct evaluation supersedes thinking about the purpose of conducting an evaluation. This practical leap in logic is not uncommon for most people beginning to think about evaluation, especially when it is not their primary job function. But it is not unreasonable to ask, “Why am I doing this?” Without a clear reason for the evaluation or an intended use by an end-user, performing an evaluation may feel like a huge undertaking. Focusing on the purpose and scope of the project at the outset will lead to stronger evaluation questions and approaches and, ideally, better use and application of the results by staff.

What follows is a brief overview of how to develop and define an evaluation question, and how to consider the scope of an evaluation project. Included is a series of examples on small, medium, and large-scale studies using a museum preschool program to model the different stages of the process. Developing questions and getting a sense of project scope is not entirely linear; 
you may find that you have to work backward and forward between questions, information, and resources to arrive at something that seems manageable.

\section{Defining the Question and Purpose}

Getting started with evaluation requires planning that includes answering some basic questions:

- What do I want to know?

- Who else wants to know? Do they want to know something else?

- How am I going to use the information?

The purpose of your evaluation — what you want to know- - will help to give direction to the evaluation overall. Having a clear and focused question will help to improve efforts, contribute to decision making, provide information and background, and support the goals and outcomes for a project. Knowing what to ask is related to your knowledge of the project and can help inform your strategies. The evaluation question can relate to things like: program or exhibition outcomes or overall impact, implementation and process, context and structures of a program or exhibition, or needs and resources. Identifying stakeholders—-who else wants to know—can aid in shaping the evaluation questions to include important information for everyone who is interested in the project. Describing the function of the evaluation results-how you are going to use the information - will influence the ways in which the information can inform and shape the direction of the current project and can be useful for future projects.

\section{Examples of over-arching evaluation questions}

Evaluation questions might attempt to describe experiences, compare programs or exhibitions against goals or frameworks, or look at causes and effects between program activities and end results. The more complex the questions, the more difficult the evaluation can be. 
Description based questions are frequently less complex to undertake and can usually be answered with available resources. Questions that look to compare or measure cause and effect will require more extensive and complex evaluation processes.

Questions that describe will provide information such as:

- Who participates in our program and why?

- What information do participants already know? What do they want to know?

- What activities were most successful?

- What are the strengths and weaknesses of the program?

- When did the program attract the most visitors?

- How did participants use the materials?

Questions that compare will help explain what "is" in relation to what "should be":

- To what extent did the project achieve its goals?

- Are we reaching all the audiences we intended?

- How well does the project support the needs of participants?

Questions that look at cause and effect can help to explain the important features of a program:

- Did participants increase their understanding of the topic?

- To what extent do participants change their behaviors after the program?

For each of these broad, over-arching questions, it is useful to generate sub-questions that can further explain and define key terms and concepts.

Once the basic idea for the evaluation is framed in the form of a question, the next step is beginning to consider what information will be needed to answer the question. Defining the key concepts the evaluation will cover, and knowing what those concepts look like in practice, are 
the next major steps in defining a good study. How will you know when you see the answer to your question? These indicators are some kind of observable or collectible information or measurements that can answer the question and demonstrate success. When the question, information needed and indicators are in place, then you can begin to design the data collection instruments, collect, analyze, and report on the data.

\section{Planning for Scope}

It is important to remember that you do not need to evaluate every aspect of a program or exhibition. The purpose of defining the question is to help set boundaries for the process. Just as a program or exhibit will never explain all there is to know about a certain topic, neither can an evaluation. It may be helpful to think of evaluation projects in terms of their scope: is the evaluation small, medium, or large? The size will depend on the question, the extent and complexity of information you need, how you decide to collect the information, who collects or can collect the information needed, and how you want to analyze and report on the results. [Insert Figure 1 about here]

\section{Case Study: Evaluating a Museum Preschool Program}

To illustrate this example, let’s look at a museum preschool program. The program is geared toward children ages 2-5 years old with an accompanying adult. The broad goals of the program are to introduce young children to the world of art, imagination, creative expression, and critical thinking. Each month the museum offers different thematic focus in hour-long classes that incorporate artwork, hands-on activities, stories, music, and movement. Staff working with this program may have many different kinds of questions related to the overall success of the program, as well as what works and what doesn't in the implementation of 
activities. Planning for an evaluation project for this preschool program can vary based on the kinds of questions the staff might want to know.

A small scale project focuses on a single question and might be something that one person can carry out on their own using readily available information. For example, a staff person might want to know which programs generated the most participants, or how families used the museum following their participation in the program. Identifying the information to be collected in this example is also straightforward and based on information that is likely already part of the overall program management system. Staff can compile attendance numbers over time and ask basic questions of participants related to their interest in the topic, and their use of the museum before and after participating.

A medium scale project is something compact and usually bounded by a timeframe or particular format. In the case of the preschool, a medium sized evaluation might focus on a full year of programmatic activities. This could include an overarching question that looks at the overall strengths and weaknesses of the curriculum, or might focus on a specific component of the project, such as adult participation over the course of the year's offerings. In a medium scale project, the evaluation activities span across multiple interactions with participants. Program staff may need some assistance from those with additional training to design more complex data collection instruments, or simply more assistance in collecting and analyzing data. The boundaries of a project help to keep the information needed within manageable limits. In this case the program timeframe and focus on specific activities keeps the focus on just those aspects of the program.

Large scale evaluation projects are those that include broad more complex questions with multiple components. These types of studies might span longer time frames, multiple formats, or 
systems. This type of project often requires more staff to implement and frequently requires the expertise of an on-site evaluator, or sometimes, an external evaluator. A large scale evaluation question for the preschool might look at the preschool children's participation in the museum programs over time and measure a variety of long-term outcomes in terms of developmental skills, and impact of their success in school as a result of their participation. A large scale evaluation might emphasize a close look at the idea of critical thinking in the program and the extent to which the program's activities can demonstrate a measurable difference in children who participated in the preschool versus those who did not.

\section{Are you in over your head?}

Having a good handle on the scope of the evaluation can help you know if you are taking on too much or if you need extra help. The complexity of the project will also dictate to some degree the level of expertise that is needed to carry out the project successfully. A common approach in planning an evaluation, especially when you have limited staff or limited resources, is to create an internship around it. As with any project, the appropriate knowledge base is crucial, and it should stand to reason that if you don't know how to do the evaluation, an intern is not likely to know either. Trained interns with a general knowledge of programs can be supportive in collecting data and to some extent doing the data analysis, but more extensive experience in program evaluation should be expected for any intern who is designing data collection instruments. Similarly, many staff have evaluation expectations as part of their job responsibilities. Most education staff should be able to identify questions and design the methods to carry out small scale studies relatively easily; designing data collection instruments may require more expertise at the medium and large scale level. Working with in-house or external evaluation is often necessary for medium and large scale projects. In part, the external evaluator 
is positioned to be somewhat more objective about projects and will bring additional expertise in methods and resources to carry out large projects.

The good news is that evaluation practices are becoming more and more supported through various professional organizations and development opportunities. The wealth of resources available online provides museum staff with example of existing evaluation studies, tutorials, tip-sheets, and instrument examples. These resources are a great start for building your knowledge base and familiarity with planning processes and tools. When coupled with a good sense of what you want to know and what you plan to do with the results, your evaluation project can be a powerful and positive experience. 